\title{
Identification and Estimation of Non-Permitted Food Colours (Sudan and Rhodamine-B Dye) In Chilli and Curry Powder by Rapid Colour Test, Thin Layer Chromatography and Spectrophotometry
}

\author{
Shruti Singh, Himani Shah ${ }^{*}$, Ritika Shah and Krishna Shah \\ Department of Biotechnology, Mithibai College, Vile parle (W), \\ Mumbai - 400056, Maharashtra, India \\ *Corresponding author
}

\section{Keywords}

Identification,

Non-permitted,

Rapid colour,

Chromatography

and

Spectrophotomentry.

Article Info

Accepted:

21 June 2017

Available Online:

10 July 2017

\section{A B S T R A C T}

A study was done to detect the presence of Sudan and Rhodamine-B dye quantitatively and qualitatively in total 16 chilli and curry samples collected from different locations from western region of Mumbai. Both branded as well as non-branded samples were selected for the study to determine the adulteration levels. A standard control of the Sudan and Rhodamine-B was used with a stock concentration of $100 \mathrm{ppm}$ and $1000 \mu \mathrm{g} / \mathrm{ml}$ respectively. Samples were extracted based on organic solvent extraction using chloroform for TLC, ethyl acetate and methanol for Spectroscopy. The concentrations of the extracted Sudan and Rhodamine-B ranged from 5.5-16ppm for Sudan (Sudan III, IV) and 4.5$22 \mu \mathrm{g} / \mathrm{ml}$ for Rhodamine-B by multiple point standard addition method. Out of the total 16 sample tested, $62.5 \%$ and $68.75 \%$ of the total samples showed the presence of Sudan and Rhodamine-B respectively by color test and $37.5 \%$ and $50 \%$ confirmed their presence by TLC and visible spectroscopy method. The present study indicates that there is prevalence of Sudan and Rhodamine-B used in spices. According to the regulation of various governmental agencies its use in food is strictly prohibited because of its ability to form carcinogenic compounds. We presented a simple, convenient and expeditious method which includes colour test, spectrophotometer followed by thin layer chromatography technique and the results were compared to multiple point external standard methods. This study was an attempt to bring in awareness among the people on the important subject of food adulteration and various simple methods available to detect food adulteration.

\section{Introduction}

Synthetic food colours are used worldwide to avoid the loss of original colour in processed foods, as well as to make the products more attractive to consumers. Synthetic food colours are considered superior to natural food colours in terms of their colour value, uniformity, and applicability in various processed foods. Synthetic food colours have been authorized and regulated for use in food additives in many countries (Ministry of
Health, Labor and Welfare, 1947; European Council, 1994; U.S. Food and Drug Administration, 2004). Only eight coal-tar food colours are permitted in certain food products under the provision of Food Adulteration Act (1954). They have included three Red shades namely, Carmoisine, Ponceau 4R, Erythrosine, two yellow shades Sunset yellow-FCF and Tartrazine, two blue shades Brilliant Blue FCF and Indigo 
Carmine and one green shade i.e. Fast green FCF. However certain unpermitted colours such as Metanil Yellow, Rhodamine B (RB), Orange G, Pararosaniline (PA), Auramine O (AO), Sudan dyes, Blue VRS and certain oil soluble colours are often added in foods as adulterants.

Sudan dyes (Sudan I, Sudan II, Sudan III and Sudan IV), Sudan red B, Sudan red7B, Sudan red G, Sudan orange G, Sudan black, Dimethyl yellow, Parared are familiar compounds in the class of azo dyes that are usedfor different industrial and scientific applications. They are widely used in industrial applications and research. They have been used in paints and cosmetic products (Cornet V et al., 2006, Anderton MS et al., 1997). Scientific applications include staining of lipids with Sudan IV for visualization and analytical purpose. Sudan dyes are however illegally used as food adulterants, particularly in red chilli powder and in chilli containing foods such as curry, frozen meats and spice mixes because of their intense red-orange colour and low price. However Sudan I-IV dyes as food additives are not permitted worldwide due to their carcinogenicity. Even though Sudan dyes are banned the European Union Rapid Alert System for food and feed have reported at least 20 cases each year of Sudan I and IV (there were fewcases of Sudan III in 2006) discovery in certain food products that are imported from outside EU. Reports were mainly from Germany, UK, Greece and Italy. Often Sudan I and IV were found simultaneously in different chilli and curry products (powders, sauces) as well as in seasonings and spice mixtures. These products originated mostly from turkey, India and Russian federations. Sudan dyes are indirect carcinogens (classified as category 3 carcinogens by IARC) and are therefore banned from the use in foods in the EU (Anonymous 2009, 2005). They generate metabolites that are converted to several active mutagens and carcinogens in humans (Anonymous 2009). Sudan dyes are used in cosmetic products and animal testing has found that isomers of Sudan III cause allergic reaction (International Agency for Research on Cancer, 1975).

These dyes are weak acids; hydrogen bond acceptor at position 1 in azo group is responsible for intra-molecular hydrogen bond with phenol-OH. The majority of azo dyes could be an origin of allergic reactions, eczema, skin dermatoses (Jaskot and Costa, 1994), affects the liver, the lungs, the vascocirculatory system, the reproductive system and the immune system. While dyes of aromatic structures which contain azo linkage, amino or nitro groups are cancer causing in experimental animals as well as for humans (Larsen et al., 2008, Ng et al., 1995, Nikulina et al., 1995, Przybojewska 1996).

Rhodamine is used as a dye and as a dye laser gain medium. Rhodamine is a banned dye as per PFA act (1954), by Government of India, because the same dye has found to be carcinogenic in human. It is harmful to humans because of its carcinogenicity, reproductive and developmental toxicity, neurotoxicity, and acute toxicity. It is used for food adulteration in sweets and confectionery. It causes health hazards in humans who consume it. Examination for this is been done for the detection of Rhodamine from Food Stuffs in Forensic Toxicology Laboratories and Food Adulteration Laboratories. Analysis of samples of sweets and confectionery collected during festivals showed the wide usage of non-permitted colours like Rhodamine to the extent of 10-95ppm, orange II (135-560ppm) and Auramine (15-400 ppm) (Sudershan et al., 1995). The use of RB and $\mathrm{AO}$ has been reported in several developing countries such as Malaysia, the Philippines, India, Vietnam, Argentina and China 
(Sudershan 1994). In Japan, the use of RB and PA in imported processed foods has also been reported (Uma et al., 1996). Thus, effective monitoring of basic colour contaminants in processed foods is necessary to ensure food safety. Rhodamine B is also proved to be carcinogenic and toxic to humans and animals (International Agency for Research on Cancer, 1978).

The US "Colours in Food Regulations" have classified Rhodamine-B as an illegal colorant and forbidden its use in foodstuffs. But there is still no regulation on the use of Rhodamine$\mathrm{B}$ in food in some developing country such as Argentina.

In China, it also has been existed in the list of illegal food additives. Unfortunately, the contamination of Rhodamine-B in condiments is still occurred under some circumstances, and the monitoring of Rhodamine-B in real samples has become a concern. Chilli powder is an important type of condiments for human being and is widely added in various foods such as pickles. Because the matrix of chilli powder sample is very complex, it is necessary to concentrate the analyse and eliminate the matrix effect. Analytical techniques have been used for determination of Rhodamine-B, like high pressure liquid chromatography, capillary electrophoresis, UV-visible spectrophotometry, and fluorimetric determination.

Although chromatographic separation enables more sensitive quantitative analysis, it may not be accessible in many laboratories because of the high price of the apparatus and professional operators (Bachalla, 2016).

UV-visible spectrometry is an attractive method due to its simplicity and lower cost than other apparatus. Quantitative UV detection of Rhodamine-B in real samples requires a sample-preparation technique that can isolate and concentrate target analyte before determination.

The technique is also suitable for routine analysis as it is characteristically known for its simplicity, rapidity and cost effectiveness.

Although various analytical methods have been developed for the detection and determination of basic colorants in processed foods such methods have several drawbacks, including time-consuming steps, a lack of application data to various processed foods, or unavailable data for the determination of low levels of basic colours. Near infrared (NIR) spectroscopy, vibrational spectroscopy techniques and Raman spectroscopy were examined by scientist (Alim et al., 2015) for the detection of Sudan dyes in various food stuffs. Capillary electrophoresis and gas chromatographic methods have also been reported. Due to expensive and time consuming chromatographic methods others methods and techniques are also developed to check and detect Sudan dyes (Chiye et al., 2014; Sana et al., 2013).

The objective of the present study was to detect the presence of Azo dyes (Rhodamine$B$ and Sudan dye) quantitatively and qualitativelyin chilli and curry powder samples collected from local market of western Mumbai city by a simple rapid colour test and visible spectrophotometric method followed by TLC (thin layer chromatography).

\section{Materials and Methods}

In the present study we determine the presence of various non-permitted food colours in Chilli and curry samples (Fig. 1) using preliminary colour test, thin layer chromatography and UV spectrophotometric analysis. The study methods are given by the following procedures and the test was done in 
the laboratory with both the qualitative and quantitative analysis.

Spices samples collected for testing the presence of non-permitted colours (Sudan III, IV and Rhodamine B)

In the present work initially, a market survey was carried out and all the 16 samples (chilli powder and curry powder, paste) consisting of both branded and non-branded, were purchased from different shops of western Mumbai, India during the period of 20162017 which is mentioned in the following table 2 .

Collection stations were chosen from different locations of western Mumbai to get a true picture about the adulteration of food or food items with Sudan and Rhodamine. From each place 50 grams of the samples was collected. Fresh chilli, dry chilli fruit and standard (Sudan III, IV and Rhodamine B) were also used and tests were performed for comparison.

\section{Colour tests}

Colour tests are based on colour change reactions in the presence of specific reagents such as conc. Hydrochloric acid, Conc. Sulphuric acid, Carbon tetrachloride, Hexane, etc. The colour change reactions of synthetic food colorants in the presence of specific reagents are shown in the following table 1:

\section{Thin layer chromatography}

\section{Preparation of chilli samples}

5 gram of each chilli and curry samples were dissolved in $20 \mathrm{ml}$ chloroform and were placed on the rotatory shaker overnight. After 18 hours the samples were removed from shaker and filtered. The filtrate was further used for thin layer chromatography.

\section{Preparation of standard solution}

Stock solutions of Standards were prepared by dissolving $2 \mathrm{mg} / 50 \mathrm{ml}$ of solvent (chloroform) and stored at $10^{\circ} \mathrm{C}$. The working standard solution was obtained from the stock by dilution (1:5) with the solvent at the time of analysis.

\section{Preparation of TLC plates}

TLC plates $(15 \mathrm{~cm} \times 7 \mathrm{~cm})$ was prepared with the help of silica gel slurry which was prepared in distilled water and was applied as a $0.25 \mathrm{~mm}$ thin layer. It was allowed to dry and was then activated in oven for 1 hour at $110^{\circ} \mathrm{C}$

\section{Experiment}

Samples and standards are applied as spots using capillary on the prepared TLC plates. The development of plates was performed in a TLC jar with benzene, hexane and acetic acid (40:60:2) for Sudan and 2-butanol, methanol and sodium sulphate (1:1:1) for Rhodamine as the solvent system for 1 hour.

For visual evaluation the chromatograms were evaluated under normal light and the distances moved by the solvent and the spots were measured by template scale and their $R_{\mathrm{f}}$ values (distance travelled by the sample in relation to the distance travelled by the solvent) was measured, calculated and compared with the standards (Alim et al., 2015)

\section{UV spectrophotometric analysis}

\section{Standard addition method}

In the present work, Standard addition method is used for the spectrophotometric determination of Sudan dyes. 
Standard addition method can be applied to most analytical techniques and are generally used to solve the matrix effect problems. In this method multiple point are adopted.

\section{Multiple point standard addition}

The method involves the construction of a calibration curve (Fig. 2) to determine the concentration of an analyte in the unknown sample by comparison to a set of samples of known concentration (standards) (Sana et al., 2013)

\section{Extraction and sample preparation of chilli for Sudan/Rhodamine}

2.5 grams of chilli powder samples were accurately weighed and transferred into a 30 $\mathrm{mL}$ centrifuge tube containing $20 \mathrm{~mL}$ of ethyl acetate and $20 \mathrm{~mL}$ of methanol for Sudan and Rhodamine respectively. The mixture was blended with the homogenizer at $16,500 \mathrm{rpm}$ for 5 minutes and was then centrifuged for 5 minutes. After centrifugation upper organic layer was filtered and collected in a $25 \mathrm{~mL}$ volumetric flask and was used to take the absorbance.

\section{Preparation of standards of Sudan I-IV dye}

Standards of each Sudan dye in concentration range of 0.01-30 ppm were prepared from Sudan stock solution of $100 \mathrm{ppm}$.

\section{Preparation of standards of rhodamine dye}

Standards of each Rhodaminedye in concentration range of $1-50$ microgram were prepared from Rhodamine stock solution of $1000 \mathrm{microgram} / \mathrm{ml}$.

\section{The experimental work}

Visible spectrums of the standards were recorded in the wavelength range of 400-700 $\mathrm{nm}$. The $500 \mathrm{~nm} \lambda$ for Sudan III, Rhodamine and $520 \mathrm{~nm} \lambda$ for Sudan IV were selected from the spectra due to their maximum absorbance at the respective wavelengths. Finally the absorbance of each sample was measured for the presence of the dyes at these wavelengths (Table 2).

A graph was plotted for absorbance Vs concentration (ppm) for standard, and the absorbance of each sample was extrapolated and the unknown concentration of Rhodamine and Sudan in the samples was determined.

\section{Results and Discussion}

We have examined 16 chilli and curry samples in total from different locations of western Mumbai to determine the percentage of Rhodamine and Sudan dye, the nonpermitted food colour present in different food products. According to the observations based on preliminary colour test mentioned in table 2, 10 positive samples were obtained for Sudan dye and 11 positive samples for Rhodamine B out of the total 16 samples that were analyzed (i.e. the $62.5 \%$ and $68.75 \%$ of the total samples(Table 2) contained Sudan and Rhodamine respectively) as shown in (Figures 3-5).

The results obtained for chilli and curry samples after thin layer chromatography were expressed in terms of $R_{f}$ (retardation factor) values in table 2 . The $R_{f}$ values of standards are also given in table 2 . The samples were checked by internal standard procedure and compared with the standards. It was found that Sudan dyes were present in 6 samples and Rhodamine B in 8 samples out of the total 16 samples analyzed i.e. the $37.5 \%$ and $50 \%$ of the total samples for Sudan and Rhodamine B respectively (Table 2, Figs 6 and 7) showed similarities with standard by comparing the chromatograms. Other samples which showed coloured spots but did not match with any of the standard $R_{f}$ may contain other types of azo 
dyes or food colorants (Bachalla, 2016; Purba et al., 2015; Alim et al., 2015). In this research work a conventional classical instrumental spectrophotometric method is described and used for the estimation of Sudan and Rhodamine B dyes. The Spectrophotometric method seems to be adequate for the detection of banned Sudan dyes (III, IV) and Rhodamine B.

To assure the validity of the results obtained limit of detection and limit of quantification were also determined. For this purpose pure chilli powder sample which was not adulterated was used with the standards.

The statistical approach adopted for the estimation of dyes is multiple point standard addition method (Calibration Curve method) (Fig. 3) for spectroscopic analysis.

The standard addition method is preferred over external standard method because pure chilli sample contains certain type of carotenoids that may interfere in the analysis in the same wavelength regions where Sudan dye appears. So, to avoid false positive result and to nullify the effect of matrix, standard addition method is used. The results obtained by this approach prove the reliability of data.

Ethyl acetate and methanol was used for extraction of dyes from chilli samples and to prepare the solutions. The use of Sudan dyes and Rhodamine B as food additives is very common in our country and it is used in food products as colorant. The dye is very harmful and its use for human consumption is prohibited due to their carcinogenicity (cancer producing capacity). Red chilli powder containing Sudan dyes are strictly banned and under no circumstance should it be sold in the market. Therefore, we developed a simple, convenient and reliable method to estimate these dyes. Spectrum of pure dye standards was also recorded to monitor the wavelength at which the absorbance of each Sudan dye was Maximum. Spectrum of sudan III and rhodamine shows $\lambda_{\max }$ at $500 \mathrm{~nm}$ and spectrum of Sudan IV dye shows $\boldsymbol{\lambda}_{\max }$ at 520 $\mathrm{nm}$. These wavelengths were used to monitor the concentration of each dye in samples.

Among the sixteen collected samples the following chilli powder samples NB C3,C4,C5,C6,C7,C8 show maximum concentration of Sudan III and IV dyes and while B-C2, NBC1, C2, C4, C7, C8, BC-CP4, NB-CP1 chilli and curry powder samples show maximum concentration of Rhodamine dyes.

The other samples which showed positive result for the colour test may contain other types of azo dyes or other food colorants. The ranges of concentration are 5.5-16 ppm for Sudan III, IV and $4.5-22 \mu \mathrm{g} / \mathrm{ml}$ for Rhodamine by multiple point standard addition method which is more than the permissible limit and quoted by other authors also (Sana et al., 2013; Alim et al., 2015). The overall range of concentration of Sudan dyes and Rhodamine determined in samples were same by multiple point standard addition method.

Fig.1 Chilli and Curry samples

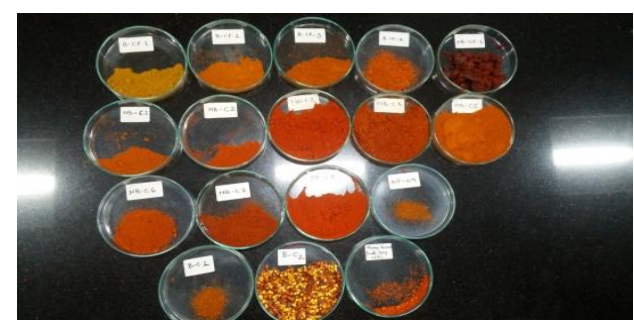


Fig.2 Calibration curve for sudan III and IV
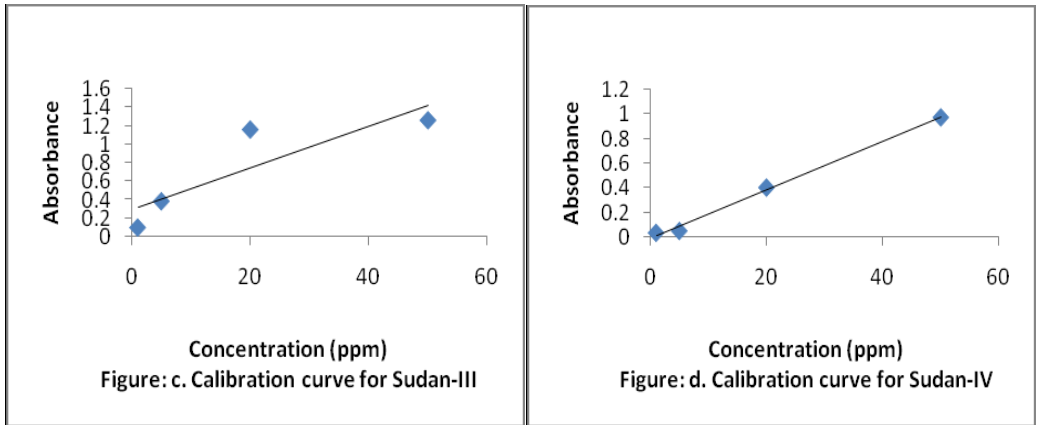

Fig.3 Chilli and curry samples showing positive results for the presence of Rhodamine B by colour test

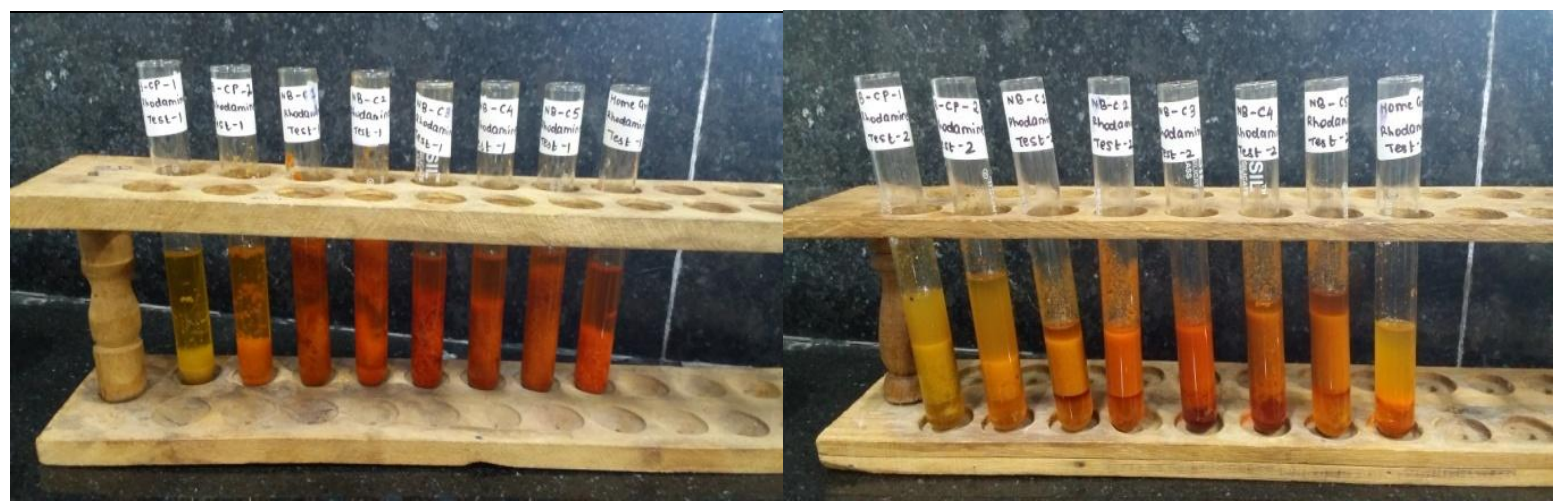

Fig.4 Chilli and curry samples showing positive results for the Presence of Sudan by colour test

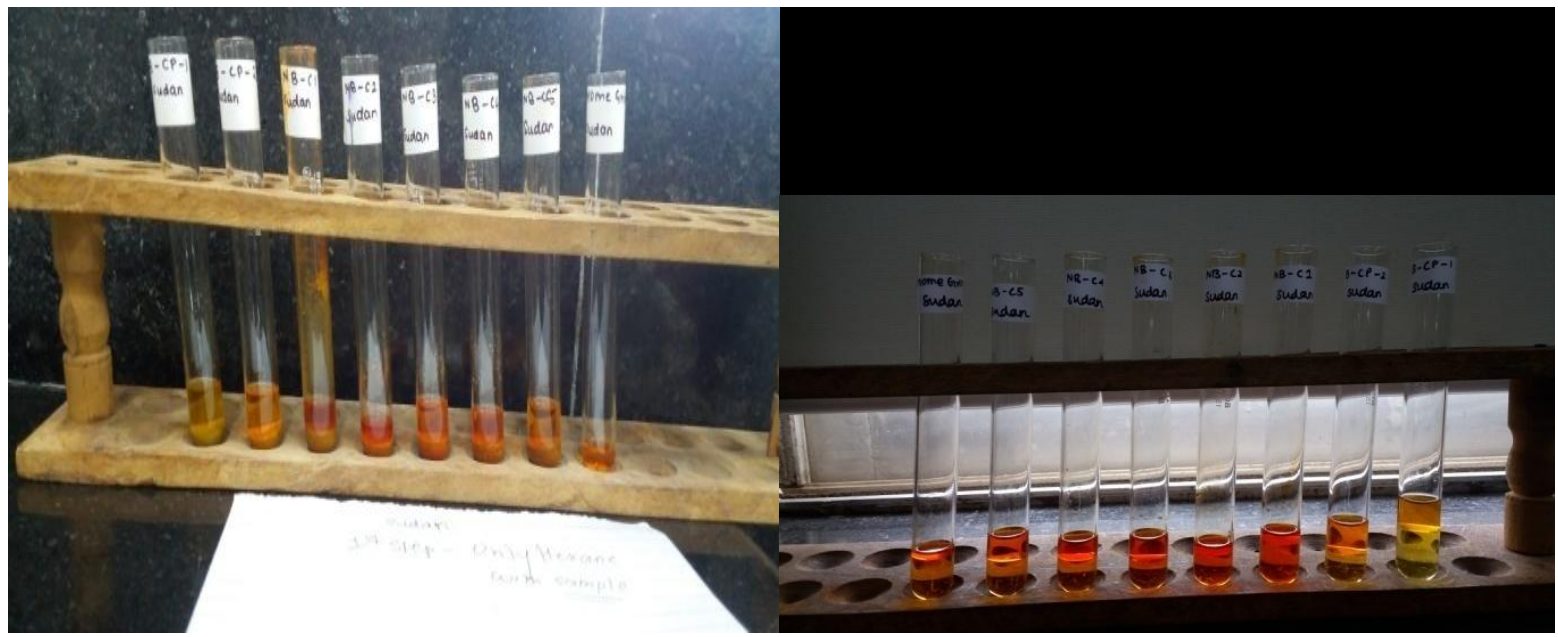


Fig.5 Chilli and curry samples showing positive results for the Presence of Sudan by color test and standard of Sudan

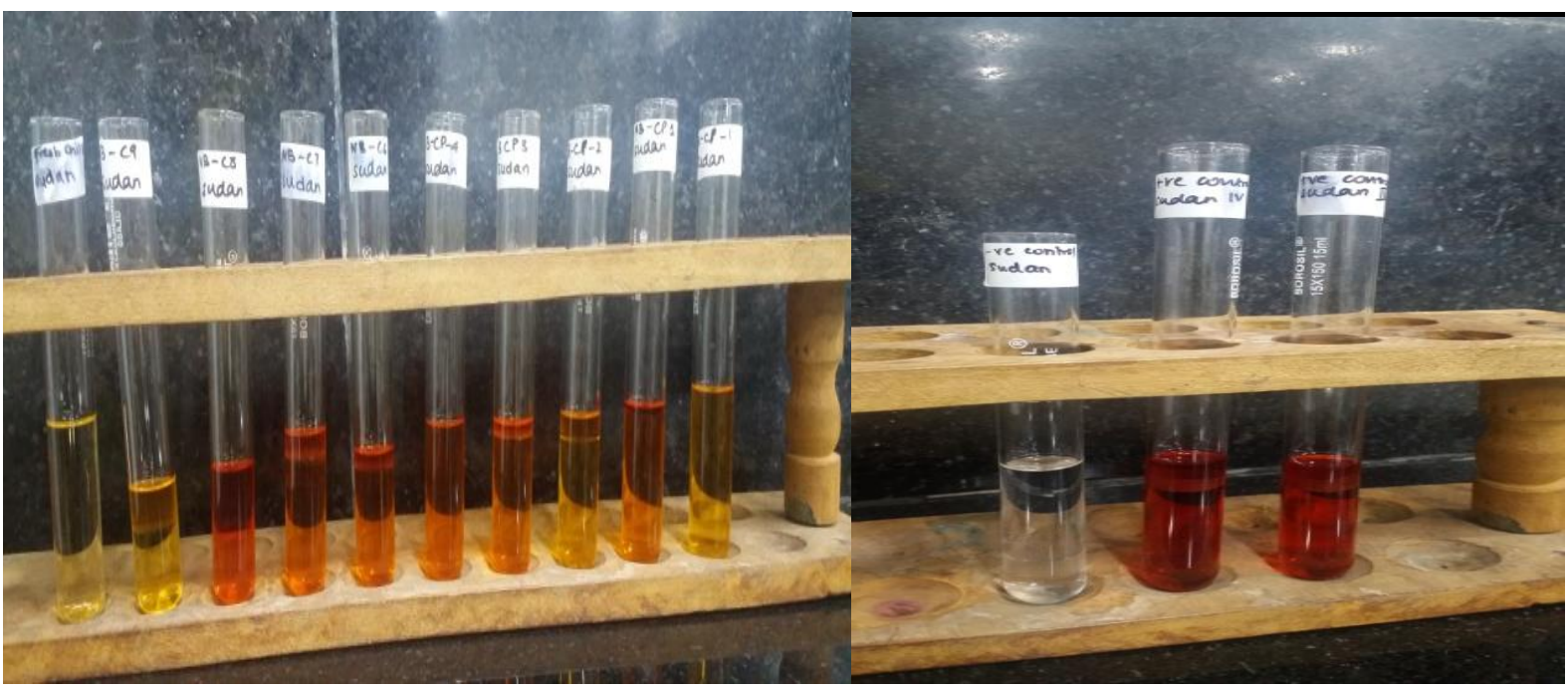

Fig.6 TLC chromatogram showing standard Sudan III, IV and Chilli samples NB-C3 AND NB-C8

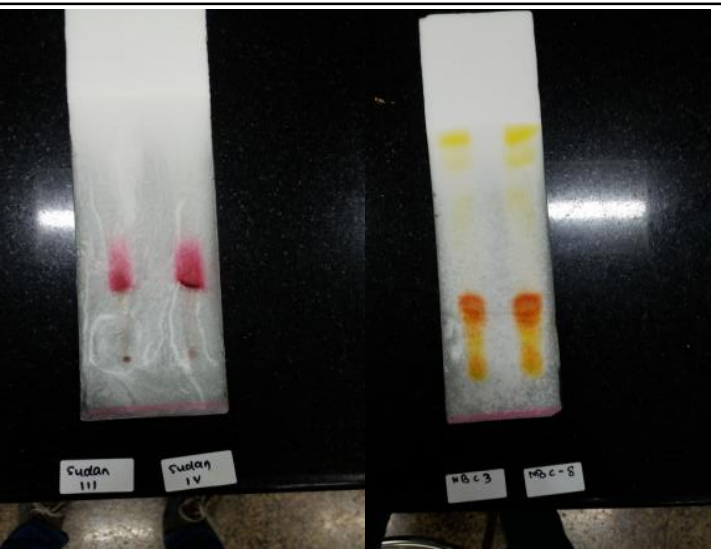

Fig.7 TLC chromatogram showing standard Rhodamine B and Chilli samples NB-C2 AND NB-C7

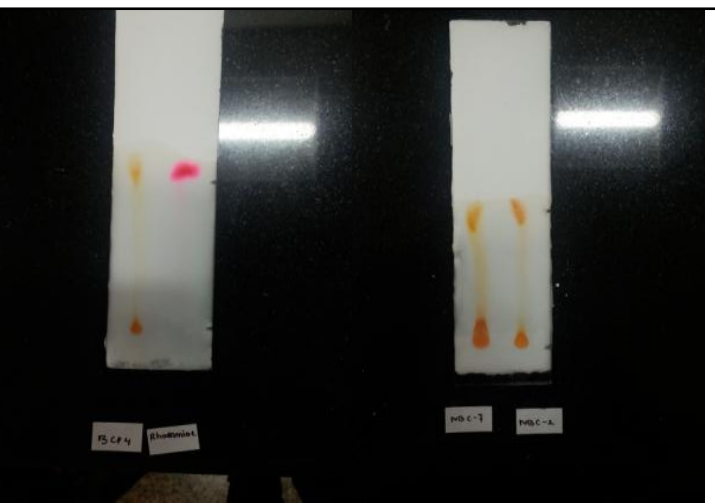


Table.1 Method for detection of common adulterant in spices

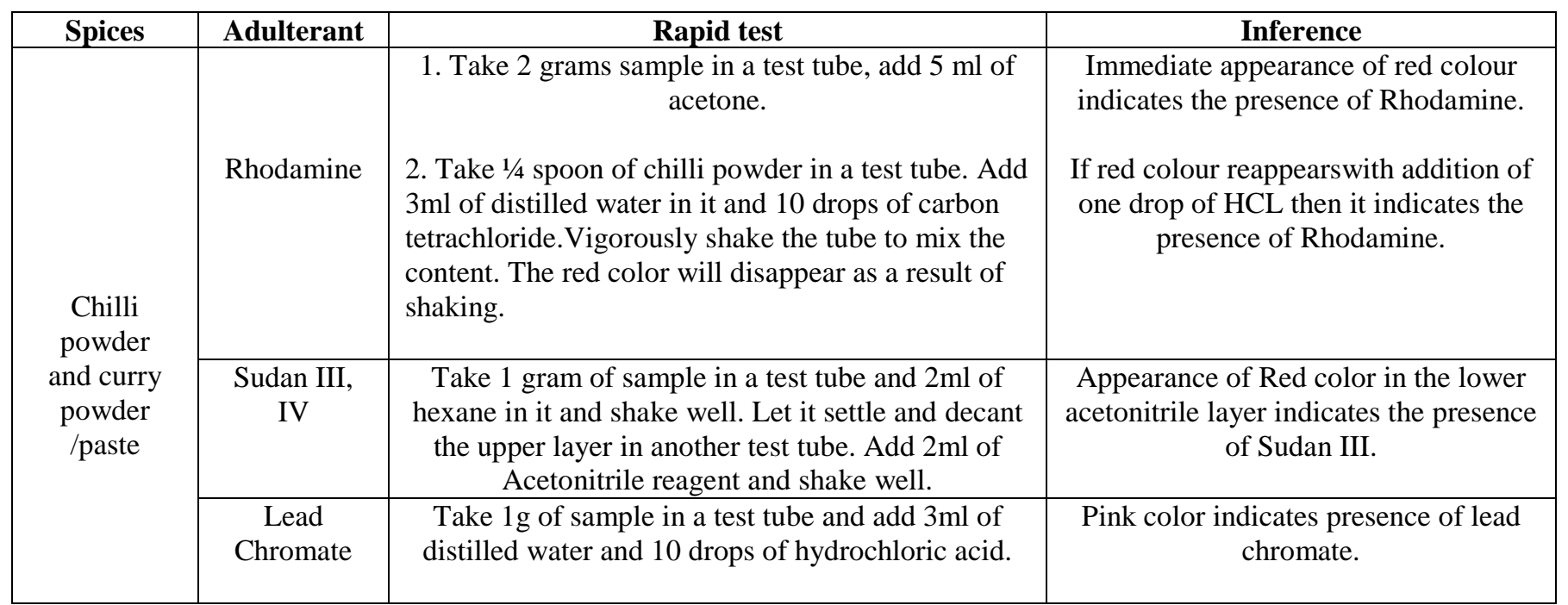

Table.2 Chilli and curry samples showing results for colour test, $\mathrm{R}_{\mathrm{f}}$ values for TLC and concentration of Sudan $(\mathrm{ppm})$ and Rhodamine B $(\mu \mathrm{g} / \mathrm{ml})$ by multiple point standard addition method

\begin{tabular}{|c|c|c|c|c|c|c|c|c|}
\hline \multirow{2}{*}{$\begin{array}{l}\text { Spices } \\
\text { Sample }\end{array}$} & \multicolumn{4}{|c|}{ Colour tests } & \multicolumn{2}{|c|}{$\begin{array}{c}\text { Thin layer } \\
\text { Chromatography }\end{array}$} & \multicolumn{2}{|c|}{ Spectrophotometric analysis } \\
\hline & Sudan & $\begin{array}{l}\text { Rhodamine } \\
\text { (Test 1) }\end{array}$ & $\begin{array}{l}\text { Rhodamine } \\
\text { (Test 2) }\end{array}$ & $\begin{array}{c}\text { Lead } \\
\text { chromate }\end{array}$ & $\begin{array}{l}\mathrm{R}_{\mathrm{f}} \text { of the } \\
\text { samples } \\
\text { with } \\
\text { respect to } \\
\text { Sudan }\end{array}$ & $\begin{array}{l}\mathrm{R}_{\mathrm{f}} \text { of the } \\
\text { samples with } \\
\text { respect to } \\
\text { Rhodamine } \\
\mathrm{B}\end{array}$ & $\begin{array}{l}\text { Concentration } \\
\text { of samples for } \\
\text { sudan (ppm) }\end{array}$ & $\begin{array}{c}\text { Concentration } \\
\text { of samples for } \\
\text { rhodamine B } \\
(\mu \mathrm{g} / \mathrm{ml})\end{array}$ \\
\hline B-C1 & - & - & - & - & - & - & - & - \\
\hline $\mathrm{B}-\mathrm{C} 2$ & - & + & + & - & - & 0.89 & & 22 \\
\hline NB-C1 & + & + & + & - & 0.32 & 0.89 & 9 & 5.5 \\
\hline $\mathrm{NB}-\mathrm{C} 2$ & + & + & + & - & 0.50 & 0.89 & 8.5 & 10 \\
\hline NB -C3 & + & + & + & - & 0.24 & 0.47 & 5.5 & 18 \\
\hline $\mathrm{NB}-\mathrm{C} 4$ & + & + & + & - & 0.24 & 0.89 & 13 & 4.5 \\
\hline $\mathrm{NB}-\mathrm{C} 5$ & + & + & + & - & 0.24 & 0.31 & 10 & 10 \\
\hline NB -C6 & + & + & + & - & 0.24 & - & 11 & \\
\hline NB -C7 & + & + & + & - & 0.24 & 0.89 & 8 & 6 \\
\hline $\mathrm{NB}-\mathrm{C} 8$ & + & + & + & - & 0.24 & 0.89 & 16 & 10.5 \\
\hline NB -C9 & - & - & - & - & - & - & & \\
\hline B -CP1 & - & - & - & - & - & - & & \\
\hline $\mathrm{B}-\mathrm{CP} 2$ & - & - & - & - & - & - & & \\
\hline B- CP3 & + & - & - & - & 0.38 & - & 9.5 & \\
\hline $\mathrm{B}-\mathrm{CP} 4$ & - & + & + & - & - & 0.89 & & 9 \\
\hline NB- CP 1 & + & + & + & - & 0.37 & 0.89 & 12 & 14 \\
\hline $\begin{array}{c}\text { Negative } \\
\text { control(water) }\end{array}$ & - & - & - & - & - & - & - & - \\
\hline $\begin{array}{l}\text { Standards (Sudan } \\
\text { /Rhodamine dye) }\end{array}$ & + & + & + & - & 0.24 & 0.89 & - & - \\
\hline Fresh chilli juice & - & - & - & - & 0.64 & 0.73 & - & - \\
\hline Home grind chilli & - & - & - & - & 0.67 & 0.72 & - & - \\
\hline
\end{tabular}

Key- B-Branded, NB-Non branded, C-Chilli powder, CP- curry powder 
The EU announced a decision (2003/460/EC) that specified the limit of detection for Sudan I and other similar dyes in the range of 0.5-1.0 ppm. Food products containing these dyes above this limit must be discarded. The decision was amended in January 2004 (2004/92/EC) and included Sudan II, III, and IV. Besides the carcinogenic metabolites of Para red, due to highly structural similarities to Sudan I, it is assumed that Para red is itself potentially genotoxic and possibly carcinogenic and hence taken into account of banned dyes (Branton 2005). The $\mathrm{R}_{\mathrm{f}}$ values of standards and the sample extracted were also compared on TLC plates. The similar values of retention factor of standard and sample on TLC plate confirmed the presence of nonpermitted dyes in the extracted sample. The method presented here is simple, expeditious and highly economical. The method does not require sophisticated instrumentation which makes it superior to the other methods described earlier for initial studies of nonpermitted dye in chilli and curry powder.

Our result depicts that unorganized food processing sectors indiscriminately use the Sudan and Rhodamine, the banned cheap synthetic dye, to adulterate the food items like chilli, curry powder etc. to promote the marketing of those food items in rural districts ignoring the possible toxic effects of Sudan and Rhodamine, on human health. This may be due to lack of administrative supervision about the adulterant induced human health hazards as provided in the Prevention of Food Adulteration Act of India (PFA, 2008). We also observed variations in the amount of Sudan and Rhodamine in the samples which contained the food colour above the maximum permissible limit.

From the literature we found that a variety of food were usually adulterated with nonpermitted colour such as Auramine,
Rhodamine B, Congo red, Orange II, Malachite green and Metanil yellow. Some information about the Sudan and Rhodamine induced health hazards have been reported discriminately in animal models (Riaz et al., 2005, Tripathi et al., 2007). So, in order to avoid the deleterious toxic effects in human physiological functions the use of Sudan and Rhodamine, should be stopped immediately. Otherwise, the people belonging to the lower economic stratum will be the worst sufferer as a result of the consumption of Sudan and Rhodamine, laden food items.

It can be concluded that the color test and thin layer chromatography are the preliminary methods for the examination of synthetic food colors. UV-Visible spectrophotometer is widely used for quantitative analysis and the data presented in various tables and graphs show the distinctive identification of adulteration of synthetic food colors. Nowadays, synthetic food colors are frequently used, therefore results of the study is found to be very useful to prevent the malpractice of synthetic food colour adulteration. The study is found to be fruitful for Forensic Chemistry and Toxicology. It has to be assessed very carefully because of the Medico-legal importance. Adulterations may lead to food poisoning. Synthetic food colour adulterations are more vulnerable even with lesser toxicity and sometimes death may also occur especially in children. This study is focused on detection of non-permitted food colours (Sudan and Rhodamine) in selected food samples. The used methods of detection can be used in the area of food quality control to detect and quantify such harmful and banned colours in edibles. It could be concluded that the methods used can be used for the detection of azo dyes without any massive instrumentation. Efficient analytical methods are required for evaluating toxicity of the adulterant, in order to determine 
whether there are synthetic dyes present in foods and whether they are permitted, to determine the levels, to confirm the presence of added dyes in foods and to check on the stability of dyes during processing and storage. Level of adulteration in edibles is still high and further researches can be conducted to detect various non-permitted colours in other food products so that some effective measures can be undertaken for improving the food quality of the products.

\section{Acknowledgments}

We would like to express our sincere thanks to our principal Dr. Rajpal Shripat Hande, Mithibaicollege, Vile Parle (W), Mumbai for his constant support and encouragement. We also wish to thank S.V.K.M for the wonderful facilities provided to carry out this under graduate project.

\section{References}

Ministry of Health, Labor and Welfare. 1947. Food Sanitation Act, Act No. 233 of December 24.

European Council. 1994. European parliament and council directive 94/36/EC of 30 June 1994 on colors for use in foodstuffs. Available at http://ec.europa.eu/food/fs/sfp/addit_fla vor/flav08_en.pdf (accessed 11 December 2013).

U.S. Food and Drug Administration. 2004. Summary of color additives listed for use in the United States in food, drugs, cosmetics and medical devices. Available at http://www.fda. gov/forindustry/coloradditives/coloradd itiveinventories/ucm 115641.htm (accessed 11 December 2013).

Cornet, V., Govaert, Y., Moens,G., Van Loco,J and Degroodt J M.2006. Development of a fast analytical method for the determination of sudan dyes in chili- and curry-containing foodstuffs by high-performance liquid chromatographyphotodiode array detection. J Agric Food Chem.54: 639644.

Anderton, M.S., Incarvito, D.C and Sherma J.1997 Determination of Natural and Synthetic Colors in Alcoholic and Non Alcoholic Beverages by Quantitative HPTLC. J LiqChromatogr R T 20: 101110.

Anonymous.2009. Spice Board of India annual report.

Anonymous. 2005. Opinion of the scientific panel on food Additives, Flavourings, Processing Aids and Materials in contact with food (AFC) to review the toxicology of a number of dyes illegally present in food in the EU. EFSA journal 264: 1-71.

International Agency for Research on Cancer, Some aromatic azo compounds. 1975. IARC Monogr. eval. carcinog. Risks hum. suppl.8: 225-241.

Jaskot, R. H., and D.L. Costa. 1994. Toxicity of an anthraquinone violet dye mixture following inhalation exposure, intratracheal instillation, or gavage. FundamApplToxicol 22:103-112.

Larsen, J.C., K.K. Norby, T.K. Reffstrup\& V.M. Beltoft. 2008. Opinion of the scientific panel on food Additives, Flavourings, Processing Aids and Materials in contact with food (AFC) to review the toxicology of a number of dyes illegally present in food in the EU 2005. J. EFSA 264: 1-71.

Ng, H.L., S. Araki, T. Tunigawa, and S. Sakura. 1995. Selective decrease of the suppressor inducer (CD4+CD45RA+) $\mathrm{T}$ lymphocytes in workers exposed to benzidine and betanaphthylamine. Arch Environ Health 50:196-199.

Nikulina, G. L., D.N. Deveikes. and G. Pyshnov. 1995. Toxicity dynamics of anionic dyes in the air of a work place 
and long-term effects after absorption through the skin. MebTrPromEkol $6: 25-28$.

Przybojewska, B. 1996. An evaluation of the genotoxic properties of some chosen dyes using the micronucleus test in vivo.Mutat Res. 367:93-97.

Sudershan, R.V and Ramesh V. Bhat.1995. A survey on veterinary drug use and residues in milk in Hyderabad. Food Additives and Contaminants. 12(5): 645-650.

Sudershan Rao, V. 1994.Analytical and Toxicological studies on selected newer food adulterants. Doctoral thesis, Mangalore University, Karnataka, India. 126-130

Uma, L.A., Sharada, G.S., Rao, M and Naik, R.N. 1996. Street foods- composition and microbiological profile. Nutrition Soc. Of India.

International Agency for Research on Cancer. 1978. IARC Monographs on the Evaluation of Carcinogenic Risk to Humans, Vol. 16, Pp. 212-231. Available at http://monographs.iarc.fr/ENG/Monogr aphs/vol1-42/mono16.pdf (accessed 11 December 2013).

Bachalla N. Identification of synthetic food colors adulteration by paper chromatography and spectrophotometric methods. IAIM, 2016; 3(6): 182-191.

Purba, M.K., Agrawal, $\mathrm{N}$ and Shukla SK.2015. Detection of Non-Permitted Food Colors in Edibles. J Forensic Res S4:S4-003.
Alim-U-N., Naseem Z and Farwa A.2015. Detection of Sudan dyes in different spices PAK. J. FOOD SCI., 25(3): 144149

Chiye T., Xining Z., Takashi O., Hiroki K., Kyoko S and Hiroshi A. 2014.A simple and rapid chromatographic method to determineunauthorized basic colorants (rhodamine B, auramine $\mathrm{O}$, and pararosaniline) in processed foods. Food Science \& Nutrition. 2(5): 547556.

Sana M.2013.Estimation of Para Red Dye in Chilli Powder and Tomato Sauces by a Simple Spectrophotmetric Method followed by Thin layer Chromatography JASEM ISSN 11198362 J. Appl. Sci. Environ. Manage. Vol. 17 (2) 177-184

Branton, G. P.2005. Opinion of the scientific panel on food additives, flavourings, processing aids and materials in contact with food on a request from the commission to review the toxicology of a number of dyes illegally present in food in the EU. The EFSA Journal 263:1-71.

Riaz, N., Khan, A. R., Aziz-ur-Rehman., Ali, S., Yasmeen, S., and Afza, N., 2009. Detection and determination of para red in chillies and spices by HPLC. J Chem Soc Pak 31:151-155.

Tripathi, M., Khanna, S. K. and Das, M., 2007.Surveillance on use of synthetic colours in eatables vis a vis prevention of food adulteration act of India. Food Control 18:211-219.

\section{How to cite this article:}

Shruti Singh, Himani Shah, Ritika Shah and Krishna Shah. 2017. Identification and Estimation of Non-Permitted Food Colours (Sudan and Rhodamine-B Dye) in Chilli and Curry Powder by Rapid Colour Test, Thin Layer Chromatography and Spectrophotometry. Int.J.Curr.Microbiol.App.Sci. 6(7): 1970-1981. doi: https://doi.org/10.20546/ijcmas.2017.607.234 